\title{
On Hydrophilicity Ageing of PP and PET Films Induced by Ultraviolet Radiation and Hydrogen Atoms
}

\author{
Yu. S. Akishev", M.E. Grushin, A.I. Drachev, V.B. Karalnik, A.V. Petryakov and N.I. Trushkin \\ State Research Center of Russian Federation Troitsk Institute For Innovation \& Fusion Research Russia, 142190, \\ Moscow Region, Troitsk, Pushkovykh str., 12, Russia
}

\begin{abstract}
The hydrophilicity ageing of polypropylene (PP) and polyethylene terephthalate (PET) films treated by nonthermal plasma usually takes over 150 days. In the present work, the accelerated ageing induced by hydrogen atoms and ultraviolet (UV) radiation with a wavelength of $\lambda=253.7 \mathrm{~nm}$ was studied. It was experimentally found that the hydrogen atoms restored the modified polymers to their original hydrophobicity very quickly (within several tens of seconds). UV irradiation of the modified PP film leads to degradation of the hydrophilic properties in 7-8 hours. The irradiation of PET film over the first three hours results in the hydrophilicity degradation, but further irradiation improves its hydrophilic properties. The analysis of the processes in the modified layer of PP and PET films during their ageing is performed.
\end{abstract}

Keywords: Hydrophilicity, induced polymer ageing, non-thermal plasma treatment.

\section{INTRODUCTION}

Non-thermal plasma (NTP) is widely used for surface modification of polymeric materials, for example, to give them adhesive, hydrophilic, hydrophobic, oleophobic and other properties [1-6]. NTP creates a variety of chemically active agents (ions, electrons, photons, radicals, excited atoms and molecules, etc.) near the surface to be treated. These active agents react not only with polymer molecules of the uppermost layer but penetrate also to a depth of several subsurface layers and form therefore a modified layer consisting of many molecular monolayers.

Until now, one of the unsolved problems is an "ageing" of the plasma treated polymer surfaces, i.e. loss in time imparted hydrophilic properties of the surfaces. In literature, the term "ageing of polymers" is used to refer to the processes of slow degradation of bulk and surface properties of polymers under the influence of external factors such as sunlight, outdoor temperature, humidity, oxygen and ozone, mechanical stress and others [7-9]. Ageing of a thin modified layer of the NTP-treated surface is much faster in comparison with the bulk ageing of the polymer. One possible reason is that the functional groups created by NTP in the modified layer are often the chromophores contributing to photodegradation.

The ageing of the modified layer depends on the polymer bulk structure and its surface morphology, the plasma treatment parameters (energy deposition, temperature, pressure and the kind of plasma gas), as well as on the storage condition of the treated films, including the presence or absence of UV radiation [10-13]. It is mentioned that a

\footnotetext{
*Address correspondence to this author at the State Research Center of Russian Federation Troitsk Institute For Innovation \& Fusion Research Russia, 142190, Moscow Region, Troitsk, Pushkovykh str., 12, Russia; Tel: +7-495-841-52-36; Fax: +7-495-841-57-76;

E-mail: akishev@triniti.ru
}

positive role of polymer chains cross-linking reduces the mobility of polar groups and polymer chains which results in stabilization of the surface layer properties. For example, in experiments $[14,15]$ it was shown that the processing of polymers in NTP of inert gases created a large number of cross-linking in the surface layer, and that the ageing time of the modified surface was significantly increased.

Unfortunately, the rapid ageing mechanism of a thin layer of the modified polymer surface still remains poorly understood. Therefore, predictive mathematical models of this phenomenon are also lacking. To create such models it is necessary to have extensive experimental data on the selective action of different active agents on the polymer surface modified by NTP.

In this paper, the change in time (ageing) of the hydrophilic properties of plasma treated PP and PET films under the influence of UV irradiation in air and in vacuum, as well as under the influence of hydrogen atoms at low pressure was studied. When choosing the experimental conditions for NTP treatment and UV processing it was taken into account that intensive and long lasting plasma activation can result not only in modification of the surface layer, but also in ablation of the polymer [16, 17] and in change of its bulk structure $[18,19]$. To avoid ablation and bulk modification of the films and to not create additional difficulties in interpreting the results, we have worked therefore with low doses of plasma and UV exposure, which were about 100 times less than those in the experiments [1719].

\section{MATERIALS AND METHODOLOGY}

The samples of PP (Celgard 240, $30 \mu \mathrm{m}$ ) and PET (Mylar ${ }^{\circledR}, 15 \mu \mathrm{m}$ ) films were used. They were $2 \mathrm{~cm}$ in width, and $20 \mathrm{~cm}$ in length. Modification of polymers was performed by their treatment with NTP generated by glow discharge in air at atmospheric pressure. The plasma source 
used for NTP treatment is described in detail in [6]. Plasma treatment time was equal to $1 \mathrm{~s}$. At this exposure time the energy per unit area of the treated surface was no more than $5 \mathrm{~J} / \mathrm{cm}^{2}$ which was insufficient for the surface etching. Nevertheless, even at such low level of energy density, plasma active particles are able to create a lot of breaks of the bonds in polymer chains which results in the formation of a non-coherent modified layer. Due to this, adhesive properties of the treated surface are reduced because the formed low-molecular compounds (fragments of polymer chains) are weakly coupled to the polymer [19]. The lowmolecular fragments can be removed easily with adhesive tape or washing of the films with ethyl alcohol in an ultrasonic bath for 3-5 min followed by drying in air [20]. Hydrophilic property retention after removal of the uppermost loosened layer proves unambiguously that the active plasma particles also penetrate (diffuse) inside the bulk of polymer.

The experiments on the induced ageing were carried out with NTP-modified films which either were washed in ethyl alcohol or not. In any case, the films were divided into three groups. Reference group number 1 was kept in air at room temperature and normal pressure; group number 2 was exposed to dosed UV irradiation in ambient air and in vacuum; group number 3 was processed by active plasma agents generated with a glow discharge in hydrogen at low pressure.

As a source of ultraviolet radiation, we used the mercury cylindrical lamp TUV-6 (Philips) with an electric power of 6 $\mathrm{W}$ and emissive power of $1.5 \mathrm{~W}$ at a wavelength $\lambda=253.7$ $\mathrm{nm}$. The choice of radiation with indicated wavelength is dictated by its ability to get photo-stimulated effects on the tested polymer samples as well as by absence of the ozone formation in air by photons of this UV radiation. Irradiated films were located at a distance of $2 \mathrm{~cm}$ from the axis of the lamp, where the calculated flux of UV photons at the film surface is equal to $F \simeq 10^{16}$ photon $\mathrm{cm}^{-2} \mathrm{~s}$ which corresponds to a flux of light energy with a density of $\simeq 10 \mathrm{~mW} \mathrm{~cm}^{-2}$. Irradiation of the films did not lead to heating. Therefore, the influence of thermal effects on the ageing processes under UV radiation can be excluded.

Experimental results revealed that PET film absorbs this UV radiation almost completely, while the PP film is practically transparent for this radiation. Photo-absorption by PET in the region $\lambda=250-310 \mathrm{~nm}$ is determined by the presence of oxygen containing groups in the chemical structure of the polymer [21, 22]. The absorption spectrum of pure PP, consists only of $\mathrm{C}$ and $\mathrm{H}$ atoms, located in the region $\lambda<160 \mathrm{~nm}$ and $\lambda>310 \mathrm{~nm}$ [23]. The almost complete transmission of radiation $\lambda=253.7 \mathrm{~nm}$ indicates that the concentration of impurity of oxygen containing groups is very low in the original polymer.

We used two modes of UV irradiation of films: by the doses of 1.5 hours with an interval of $1-2$ days between radiation sessions, and continuous irradiation over $1.5-7.5$ hours. In our experiments, either plasma treated side of the film was exposed to UV radiation or the opposite side. The irradiation was carried out in ambient air and in vacuum, i.e. in the presence and in absence of oxygen.
As a source of hydrogen atoms we used a stationary low pressure glow discharge in hydrogen at low pressure $(\mathrm{P}=$ $270 \mathrm{~Pa}$ ) and at current of $10 \mathrm{~mA}$. The discharge was created in a dielectric chamber with volume of 2.61 . Hydrogen flow was used as plasma forming gas. Flow rate of hydrogen was equal to $0.11 \mathrm{~atm} \mathrm{~s}^{-1}$. Before each experiment, the chamber was evacuated to a pressure of $1 \mathrm{~Pa}$ and then it was "flushed" over 10 minutes with hydrogen flow at operating pressure.

The glow discharge creates a large number of hydrogen atoms, which emit in lines $H_{\alpha}(\lambda=656.3 \mathrm{~nm}), H_{\beta}(\lambda=486.1$ $\mathrm{nm})$ and $H_{\gamma}(\lambda=434.1 \mathrm{~nm})$ well registered by routine spectroscopic method. The emission intensity of the discharge in the ultraviolet region $170<\lambda<350 \mathrm{~nm}$ is extremely low, but it is high enough in the vacuum ultraviolet (VUV) region $\lambda<170 \mathrm{~nm}$ (e.g. [23], there is intense atomic Lyman line $\lambda=121.5 \mathrm{~nm}$ ), which is well absorbed by practically all polymers.

The modified films were placed around the plasma column at a distance of $10 \mathrm{~mm}$ on two plates of Plexiglas non-transparent for VUV and UV radiation (Fig. 1). The films were placed on both sides of each plate (1). The film (2) facing the plasma was processed by VUV radiation and by plasma active particles; but the film (3) fixed on the shadow side was only activated by hydrogen atoms carried as a result of their diffusion.

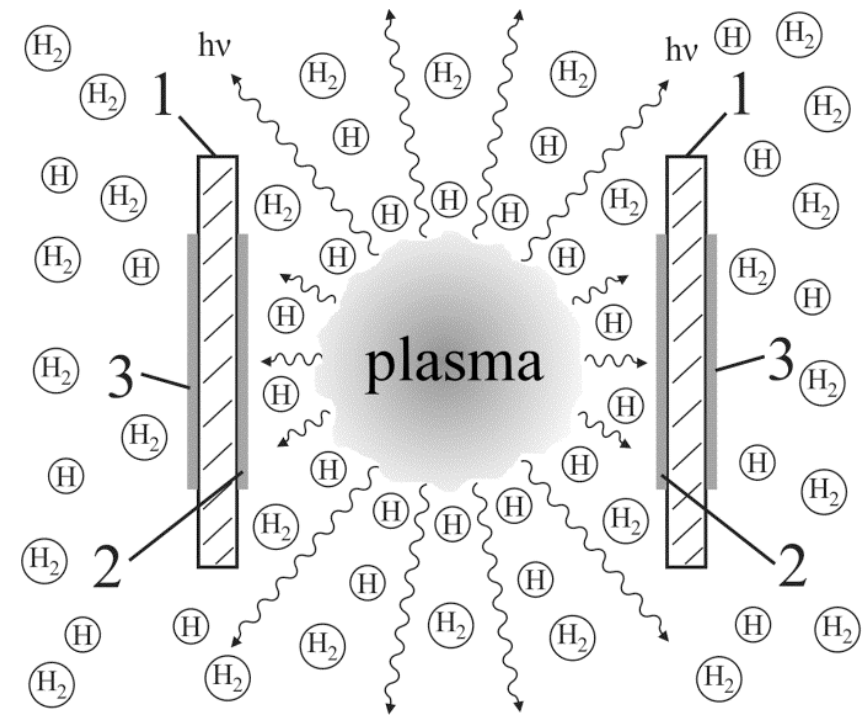

Fig. (1). Set-up for induced ageing of PP and PET films surfaces by hydrogen atoms and VUV radiation produced by plasma of glow discharge in $\mathrm{H} 2$ at low pressure: the Plexiglas substrates (1); investigated polymer films (2), (3).

The changes in hydrophilic properties of the modified PP and PET films induced by either UV photons or hydrogen atoms were characterized by goniometry method measuring the wetting contact angle for small water droplet (bidistilled water) deposited onto the film. The modified layer chemical composition was characterized by X-ray photoemission spectroscopy (XPS) using the analytical complex LAS 3000 (ISA RIBER France). The vacuum in the analyzing chamber was kept under $10^{-7} \mathrm{~Pa}$ during analysis. Excitation was via the $\mathrm{Al} \mathrm{K} \alpha$ line $(\mathrm{h} v=1486.6 \mathrm{eV})$, with the emission voltage and current of the source set to $12 \mathrm{kV}$ and $20 \mathrm{~mA}$, respectively. The value of $285.0 \mathrm{eV}$ of the hydrocarbon $\mathrm{C}_{1 \mathrm{~s}}$ core level was used as a calibrant for the energy scale. The 
diameter of the analyzed area was $8 \mathrm{~mm}$. The spectra were acquired at a take-off angle of $90^{\circ}$ relative to the sample surface. The depth of analysis was $\leq 8 \mathrm{~nm}$ for normal detection. The multiple attenuated total reflection (ATR) technique (six reflections, 500 accumulations) was used for the analysis of the functional groups in the modified surface layer. ATR spectra registration was carried out by FTIR spectrometer Bruker Equinox 55 equipped with horizontal ATR unit A537 Brucker Optics and ZnSe crystal.

\section{RESULTS}

\subsection{Characterization of the Original and NTP Modified PP and PET Films}

Table 1 and Fig. (2) show XPS-analysis and IR spectroscopy data for the original and NTP-modified PP and PET films.

Table 1. XPS Surface Characteristics of the Original and Plasma Treated PP and PET Films

\begin{tabular}{|c|c|c|c|c|}
\hline \multirow{2}{*}{ Sample } & \multicolumn{2}{c|}{ Atomic Concentrations, \% } & \multirow{2}{*}{ O/C } \\
\cline { 3 - 4 } \multicolumn{2}{|c|}{} & C & O & \\
\hline \hline \multirow{2}{*}{ PP } & Original & 97.3 & 2.7 & 0.03 \\
\cline { 2 - 4 } & Plasma treated & 83.2 & 16.8 & 0.20 \\
\hline \multirow{2}{*}{ PET } & Original & 68.1 & 31.9 & 0.47 \\
\cline { 2 - 4 } & Plasma treated & 56.6 & 43.4 & 0.77 \\
\hline
\end{tabular}

XPS data and ATR-IR spectra of the plasma treated films show that modified surface layer is enriched with oxygen containing functional groups (hydroxyl groups $\sim \mathrm{C}-\mathrm{O}-\mathrm{H}$, hydroperoxide groups $\sim \mathrm{C}-\mathrm{O}-\mathrm{O}-\mathrm{H}$, ketones $\sim\left(\mathrm{CH}_{3}\right) \mathrm{C}=\mathrm{O}$, aldehyde $\sim H C=O$, carboxyl groups $\sim(\mathrm{OH}) \mathrm{C}=\mathrm{O}$ ), as well as with groups containing double carbon bonds $C=C$ (vinyl $\mathrm{CH}=\mathrm{CH}_{2}$, transvinylene $-\mathrm{CH}=\mathrm{CH}-$ and vinylidene $>\mathrm{C}=\mathrm{CH}_{2}$ ).

Changes in the contact angle $(\theta)$ and the $\mathrm{O} / \mathrm{C}$ ratio in the modified layer during natural ageing, i.e. under storage of the film in ambient air at room temperature, are shown in Fig. (3). A rapid increase of contact angle is observed for the first 5-10 days (we call this period as first (or fast) stage of a natural ageing). We would like to direct attention to an interesting and important fact: within the limits of $\mathrm{O} / \mathrm{C}$ measurement accuracy, the deterioration of hydrophilicity is not accompanied by a decrease in the amount of oxygen in the surface layer. In other words, the surface wettability during the first stage of ageing is determined not only by the content of bound oxygen in the modified layer, but also by the presence of structures containing double carbon bonds, for example, vinyl, transvinylene and vinylidene groups.

Note that the lack of direct correlation between the magnitude of the contact angle $\theta$ and the $\mathrm{O} / \mathrm{C}$ ratio in the modified polymer layer was also pointed out in [24,25]. The slow (or the second) stage of natural ageing in ambient air occurs in 10 days of the film storage. At this stage, the modified films keep hydrophilic properties during a long time ( $\geq 150$ days), in the course of which the content of oxygen in the modified layer is slowly decreasing.

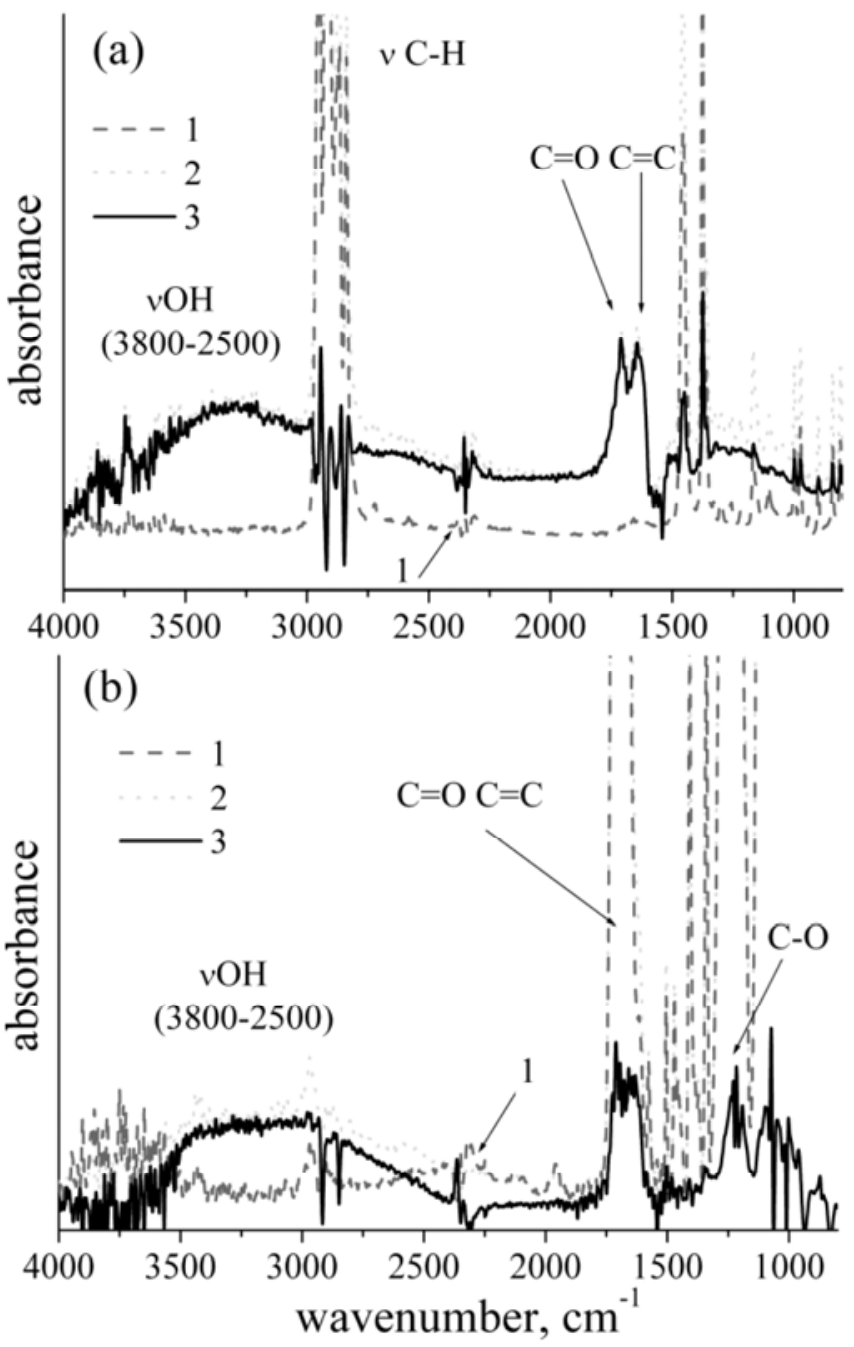

Fig. (2). PP (a) and PET (b) films ATR-IR spectra of the original film (1), plasma treated film (2) and difference spectrum of the treated and untreated films (3).

\subsection{UV Irradiation of PP Film in Air}

It was established that UV irradiation in air has no effect on the original PP film surface properties. At the same time, the UV radiation degrades the hydrophilicity of the plasma modified PP film very efficiently. The obtained results are shown in Fig. (4).

The contact angles in Fig. (4) were measured at the days marked by rhombs, measured before and immediately after UV processing of the films. The presented results demonstrate that UV irradiation of PP film in the air is able to restore completely its original hydrophobicity within a few hours. Since PP film is transparent to UV radiation, the revealed effect does not depend on a direction of radiation accelerated ageing occurs during irradiation of the film both on the side treated by plasma and on the opposite side. In addition, the increase in $\theta$ does not depend on the method of exposure (permanent or periodic with low doses) - only the total dose of absorbed light energy is of crucial importance.

\subsection{UV Irradiation of PET Film in Air}

In contrast to PP film, PET completely absorbs the UV radiation with wavelength of $\lambda=253.7 \mathrm{~nm}$. Since the 
effective length of UV absorption in PET is several tens of nanometers [22, 23], which is much less than the thickness of the used film, the irradiation influences only one side of the PET film facing the source of UV radiation.
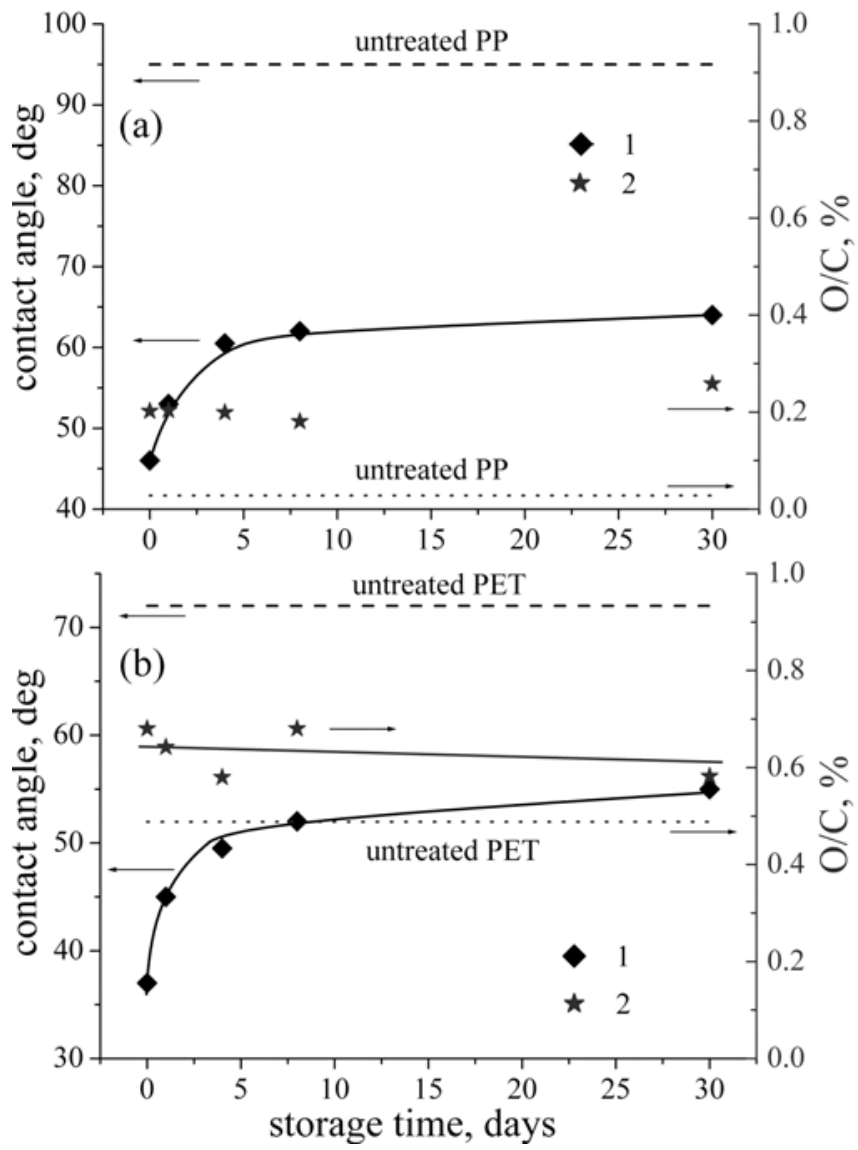

Fig. (3). The contact angle $\theta$ (1) and the O/C ratio (2) for NTPtreated PP (a) and PET (b) films as a function of storage time in ambient air.

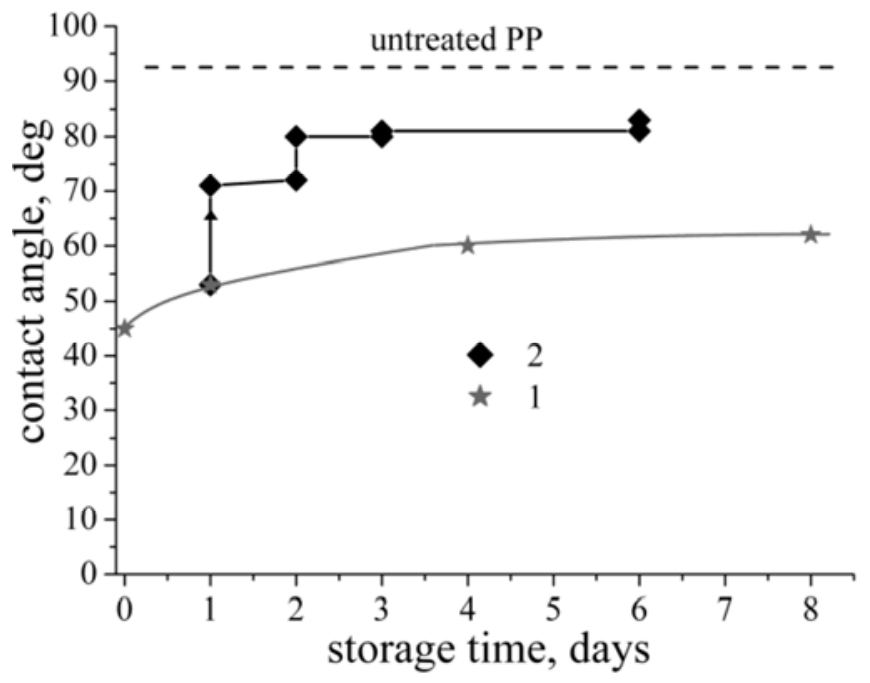

Fig. (4). Increase in contact angle of the plasma treated PP film as a function of storage time in air: (1) - without UV irradiation, (2) induced by successive doses of UV radiation; one dose corresponds to1.5 hours exposure of the film; the doses repeated in a day.

UV irradiation of the original PET film results in an increase of its hydrophilic properties (curve (1), Fig. 5). In the case of the modified film, evolution of contact angle in time under UV irradiation is non-monotonic (curve (2)): irradiation for $\leq$ three hours leads to a decrease in hydrophilicity, however the angle $\theta \approx 60$ is still smaller than the initial one (72); after 3 hours of irradiation the hydrophilicity enhancement is observed. Note that the final state of the surface after its irradiation over 6 hours is characterized by $\theta \approx 10$, which is less than that measured immediately after plasma modification of the film. Washout of loosen uppermost modified layer, enriched with structures containing double carbon bonds, leads to a monotonic improvement of hydrophilicity under UV irradiation (curve (3)).

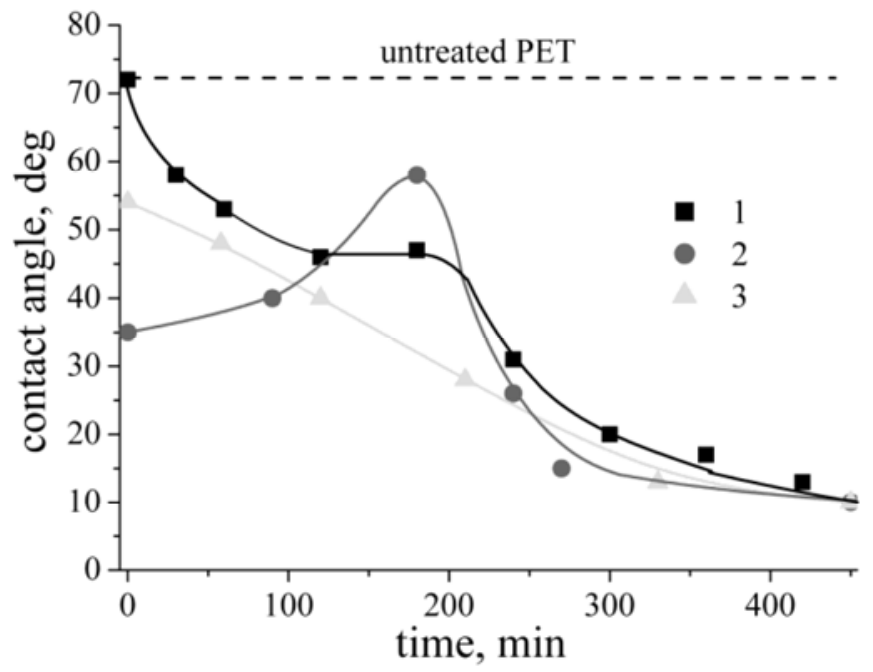

Fig. (5). The contact angle of PET film as a function of the UV irradiation time in air: (1) - hydrophilization of the original film by UV irradiation; (2) - induced by UV irradiation degradation of hydrophilicity of the plasma treated film followed by an increase of hydrophilicity; (3) - the plasma treated film hydrophilization by UV irradiation. The film was washed in ethanol prior to UV irradiation.

Thus, the experiments have shown that long term $(\geq 6$ hours) UV irradiation of the PET film in ambient air is able to create a strong hydrophilicity of its surface. It should be noted that the ultimate value of $\theta$ obtained by long irradiation is practically independent of the initial state of the irradiated film (i.e. film treated by plasma or not, washed in alcohol or not). Furthermore, it was experimentally established that the change of $\theta$ for the modified film under its UV irradiation performed during the first stage of natural ageing does not depend practically on the way of irradiation (permanent irradiation in a single experiment or periodic exposure by low doses over several days - the main importance is the total duration of exposure time, i.e. the total dose of energy absorbed by the modified surface, so that the same contact angles correspond to the same doses).

Washout in ethanol of PET films processed by long term UV irradiation in air significantly increased the contact angle from $\theta \simeq 10-15$ to $\theta \simeq 35-37$. This fact indicates that degradation of the polymer chains grows with an increase in dose of UV irradiation while low-molecular fragments are abundantly formed. As mentioned above, these fragments are weakly attached to the surface and therefore can be easily removed from the surface by washing. 
The XPS analysis results shown in Fig. (6) provide an opportunity to answer the question, which functional groups contain the bound oxygen in the PET film processed by UV radiation in air.

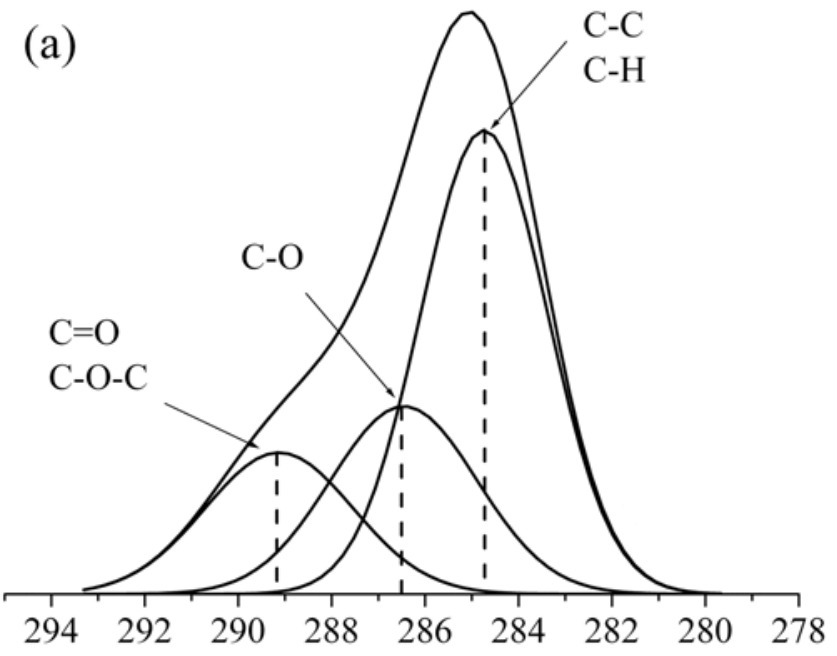

(b)

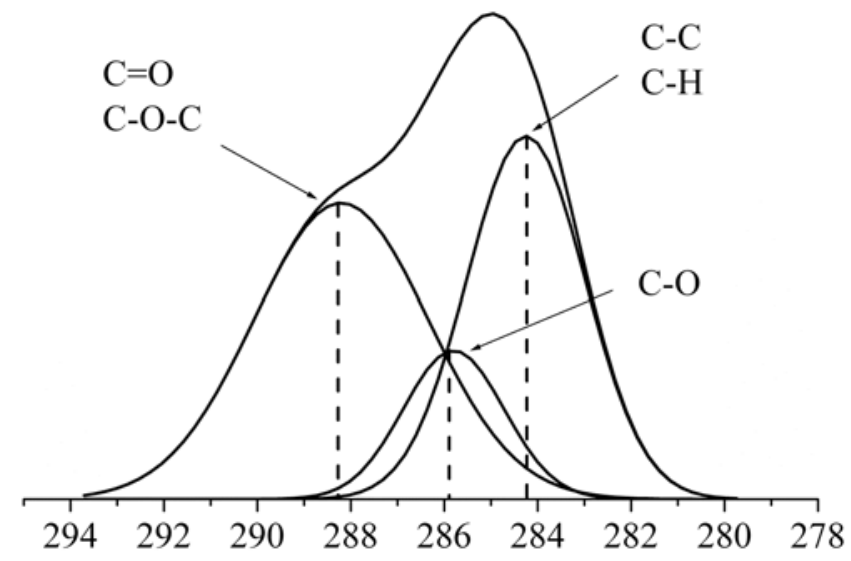

(c)

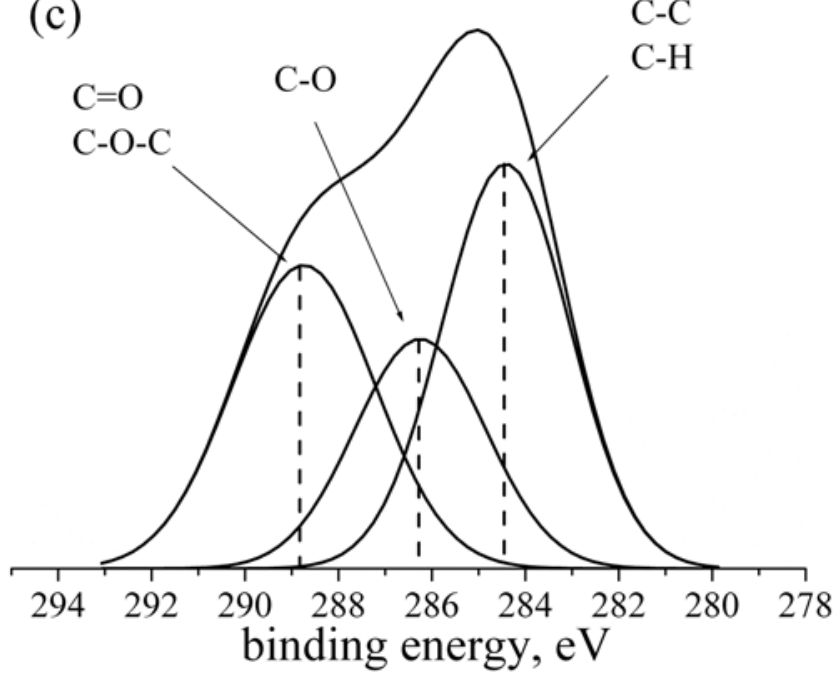

Fig. (6). The results of XPS analysis of the original (a) PET film and irradiated ones by UV light in air for 2 hours (b) and 6 hours (c).
At low dose of UV irradiation ( 2 hours) the amount of bound oxygen in the PET film increases due to the formation of functional groups containing $C=O$ (aldehydes and ketones) and $C-O-C$ bonds (esters), while the oxygen concentration in the functional groups containing $\mathrm{C}-\mathrm{O}$ bond (alcohols) is not changed practically. Another situation is observed at high dose of UV irradiation (6 hours). In this case the amount of oxygen in the functional groups containing $C$ - $O$ bonds is increased, and, on the contrary, the concentration of oxygen in the functional groups containing $C=O$ and $C-O-C$ bonds is reduced. Appropriate quantitative results are shown in Table 2.

Table 2. Changing the Characteristics of the PET Film Processed by UV Radiation $(\lambda=253.7 \mathrm{~nm})$ in Air

\begin{tabular}{|c|c|c|c|c|}
\hline \multirow{2}{*}{ Sample } & \multirow{2}{*}{$\boldsymbol{\theta}$, Degree } & \multicolumn{2}{|c|}{ Atomic Concentrations, \% } & \multirow{2}{*}{ O/C } \\
\cline { 3 - 4 } & & $\mathbf{C}$ & $\mathbf{O}$ & \\
\hline \hline Original & 75 & 68.3 & 31.7 & 0.46 \\
\hline Irradiated for 2 hours & 46 & 51.1 & 48.9 & 0.96 \\
\hline Irradiated for 6 hours & 13 & 48.8 & 51.2 & 1.05 \\
\hline
\end{tabular}

ATR-IR spectrum of the PET film processed by UV irradiation (Fig. 7) shows also that its surface layer is enriched with oxygen containing functional groups (hydroxyl groups $\sim \mathrm{C}-\mathrm{O}-\mathrm{H}$, hydroperoxide groups $\sim \mathrm{C}-\mathrm{O}-\mathrm{OH}$, ketones $-\left(\mathrm{CH}_{3}\right) \mathrm{C}=\mathrm{O}$, aldehyde $\sim \mathrm{HC}=\mathrm{O}$, carboxyl groups $\sim(\mathrm{OH}) \mathrm{C}=\mathrm{O})$, as well as with the groups containing double carbon bonds $\mathrm{C}=\mathrm{C}$ (vinyl $-\mathrm{CH}=\mathrm{CH}_{2}$, transvinylene $\mathrm{CH}=\mathrm{CH}$ - and vinylidene $>\mathrm{C}=\mathrm{CH}_{2}$ groups).

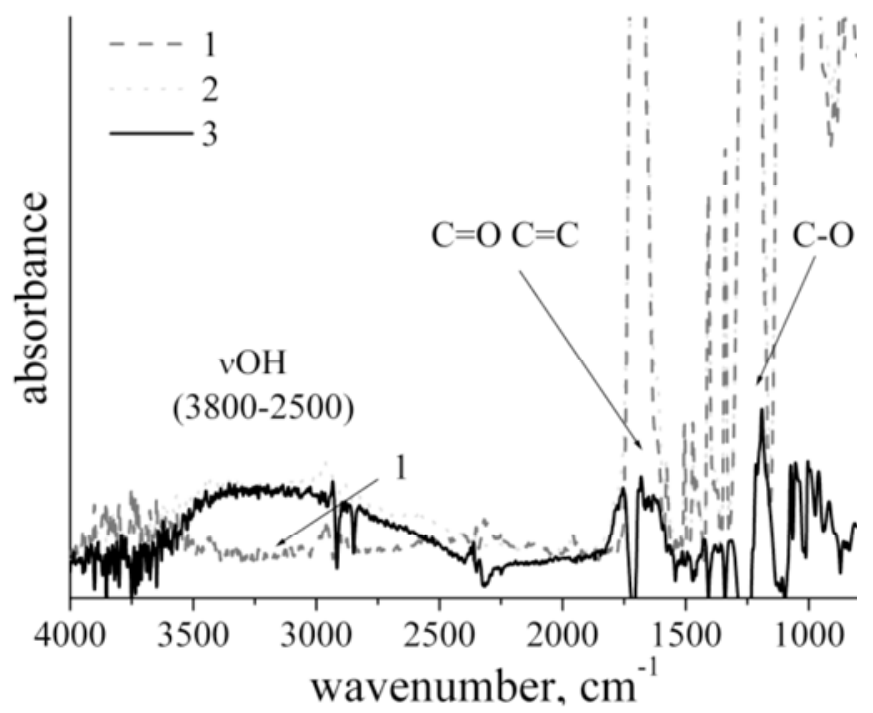

Fig. (7). ATR-IR spectra of the original PET film (1), after its processing by UV irradiation (2) in air for 6 hours and difference spectrum of the treated and untreated films (3).

\subsection{UV Irradiation of PET and PP Films in Vacuum}

The original PET film was processed by UV irradiation in chamber at low pressure (residual air pressure $P \leq 1 \mathrm{~Pa}$ ), i.e. at low oxygen concentrations $\leq 610^{14} \mathrm{~cm}^{-3}$, which is $10^{4}$ 
times lower than the oxygen concentration in ambient air). The exposure time was 2, 4 and 6 hours. The films were studied by XPS, as well as by measuring the contact angles on both sides. The contact angles were measured immediately after irradiation and in 2 days after UV treatment. After measurement of $\theta$, the film was washed in ethanol, then it was dried in air, and the contact angle was measured again. In all cases, the values of $\theta$ remained unchanged and typical for the original film $\theta \simeq 72$. The $\mathrm{O} / \mathrm{C}$ ratio did also not change within the accuracy of XPS-method used. Thus, in spite of complete absorption of UV radiation by original PET film, its hydrophilization does not occur in the absence of oxygen.

In another series of experiments a change of the plasma treated PP and PET films hydrophilicity in vacuum without UV irradiation and under UV irradiation was studied. The obtained results on the contact angle change with time are shown in Figs. $(\mathbf{8}, 9)$.

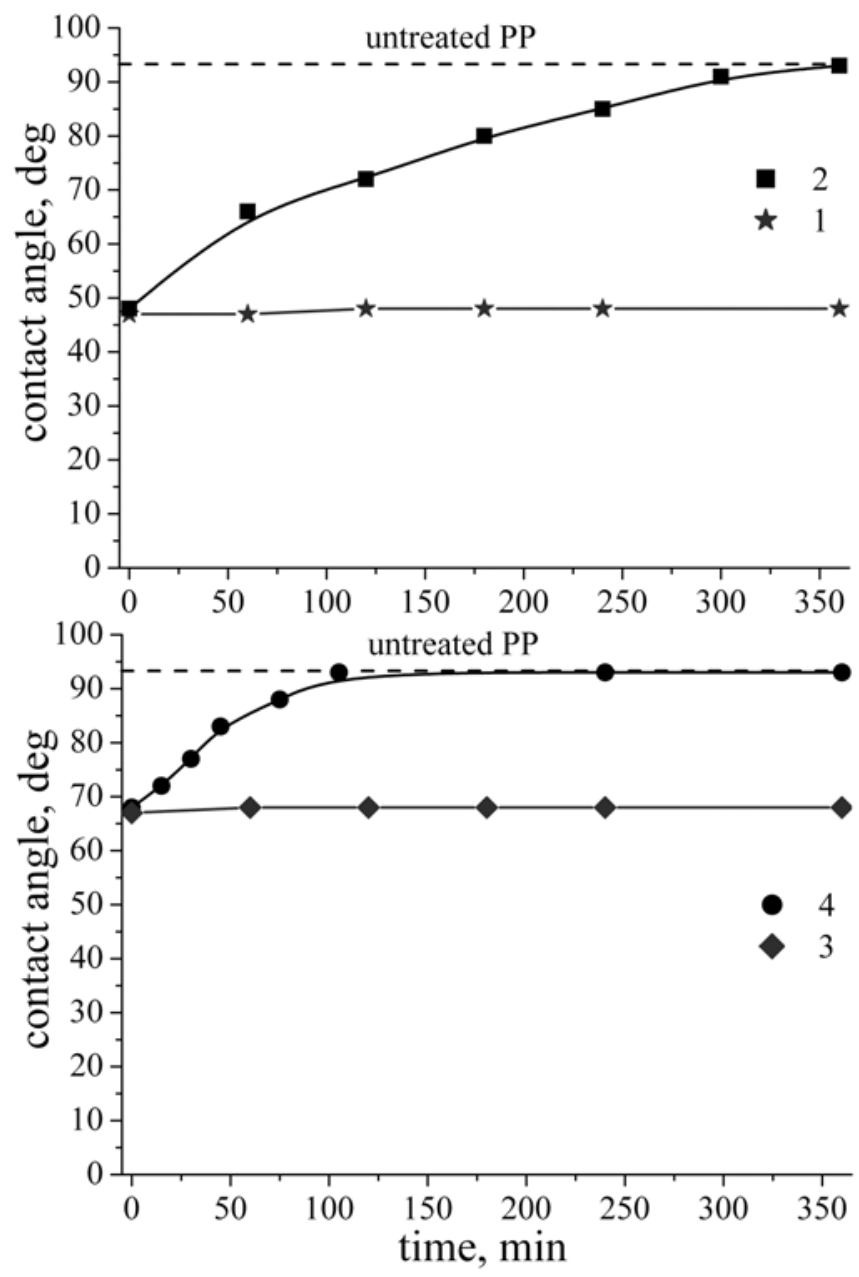

Fig. (8). The PP film contact angle changes with time in vacuum: (1) - without following UV irradiation; (2) - with following UV irradiation; (3) - washout in ethanol without following UV irradiation; (4) - washout in ethanol with following UV irradiation.

UV irradiation in vacuum leads to a rapid degradation of the hydrophilic properties provided by NTP. For the film, treated by plasma and then washed in ethanol (Figs. 8, 9, curves (4)), this process goes faster than that for unwashed film (Figs. 8, 9, curves (2)). In the case of PET film, it is revealed that in vacuum $\theta$ increases without $\mathrm{UV}$ irradiation up to the value corresponding to the washed film (Fig. 9, curve (1)). This effect can be associated with spontaneous departure in a vacuum of the low-weight products of polymer chains degradation which are weakly bound to the surface. In general, the degradation of hydrophilic properties of PP and PET films exposed to UV radiation is faster in vacuum than that in air. A comparison of the characteristics of modified PET film under UV irradiation in vacuum and in air is presented in Table $\mathbf{3}$.
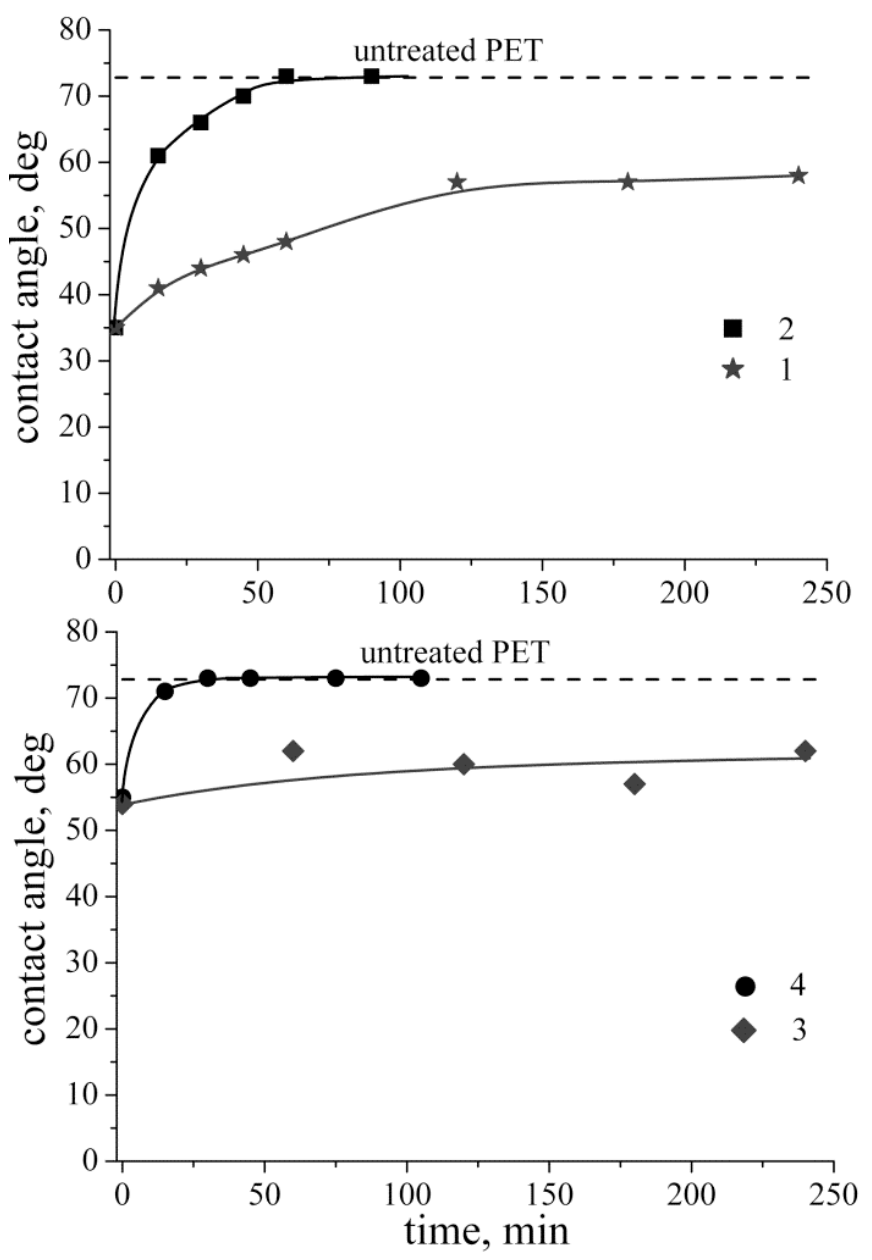

Fig. (9). The PET film contact angle changes with time in vacuum: (1) - without following UV irradiation; (2) - with following UV irradiation; (3) - washout in ethanol without following UV irradiation; (4) - washout in ethanol with following UV irradiation.

\subsection{The Modified Films Ageing Induced by Hydrogen Atoms}

Experimental results proved that the original PP and PET films did not change their hydrophobic properties under treatment in hydrogen glow discharge plasma which is characterized by high concentration of hydrogen atoms and VUV radiation (intense atomic Lyman line $\lambda=121.5 \mathrm{~nm}$ ). Contact angles of these films remained equal to the initial ones for treatment time up to 5 minutes. Thus, $\mathrm{H}$ atoms and VUV photons do not create hydrophilic properties of PP and PET films in the absence of oxygen.

A different situation is observed for the films modified by air plasma at atmospheric pressure. Fig. (10a) shows a change 
Table 3. Change of the Characteristics of the Plasma Modified Surface Layer of the PET Film Processed by UV Radiation in Vacuum and in Air

\begin{tabular}{|c|c|c|c|c|}
\hline Sample & $\theta$, Degree & \multicolumn{2}{|c|}{ Atomic Concentrations, $\%$} & $\mathbf{O} / \mathrm{C}$ \\
\hline Treated by plasma in air & 35 & 56.6 & 43.4 & 0.77 \\
\hline 3 hours of UV irradiation in vacuum & 70 & 66.2 & 33.8 & 0.51 \\
\hline 6 hours of UV irradiation in vacuum & 70 & 67.3 & 32.7 & 0.49 \\
\hline 6 hours of UV irradiation in air & 15 & 49.3 & 50.7 & 1.03 \\
\hline
\end{tabular}

of the contact angle of the modified PP film surface depending on the time of its exposure to hydrogen glow discharge environment. The film was placed on the front side of the substrate (position (2) in Fig. 1) and therefore it contacted with the hydrogen plasma and was exposed to VUV radiation. One can see (curve 1) that the PP film surface loses its hydrophilic properties extremely fast (10 s only).

The decrease of hydrophilicity of the PP film located on the back side of the opaque substrate (position (3) in Fig. 1) occurs only under the influence of hydrogen atoms, which appear behind the shadow side of the substrate due to their diffusion. In the absence of VUV radiation, more time is required to restore the original hydrophobicity - $40 \mathrm{~s}$ instead of $10 \mathrm{~s}$ (Fig. 10, curve (2)). Note that in the medium of nonactivated molecular hydrogen (glow discharge is switched off) the hydrophilicity of PP and PET films was not changed during the time typical for our induced ageing experiments (up to $30 \mathrm{~min}$ ).

The experimental results for the modified PET film are shown in Fig. (10b). Hydrophilicity of the PET film is degraded very rapidly also under the influence of the active particles of low pressure hydrogen plasma. The time needed to increase $\theta$ to the value typical for the original PET film is $20 \mathrm{~s}$, and, in contrast to the PP film, this time is practically independent of the location and orientation of the film on the substrate (see Fig. 1). This effect is possibly associated with hydrogen atoms having a determining effect on the modified PET layer compared with other active agents of hydrogen plasma, such as charged particles and VUV radiation. Extremely rapid hydrophilicity degradation under the hydrogen atoms is probably determined by fast interaction of hydrogen atoms with double $C=C$ carbon bonds resulting in a decrease of the number of unsaturated carbon bonds, as well as by detachment of oxygen from oxygen-containing functional groups to form water.

Similar results were obtained for the PP and PET films ageing induced by the active species of methane plasma. The hydrogen atoms $\mathrm{H}$ and radicals $\mathrm{CH}, \mathrm{CH}_{2}$ and $\mathrm{CH}_{3}$ formed in the methane plasma provide a significant increase of contact angles of these polymers within tens of seconds.

\section{DISCUSSION}

\subsection{On Natural Ageing of the Modified Films at the First Stage}

In principle, to adequately calculate the surface energy it is necessary to use $a b$ initio methods. Unfortunately up to

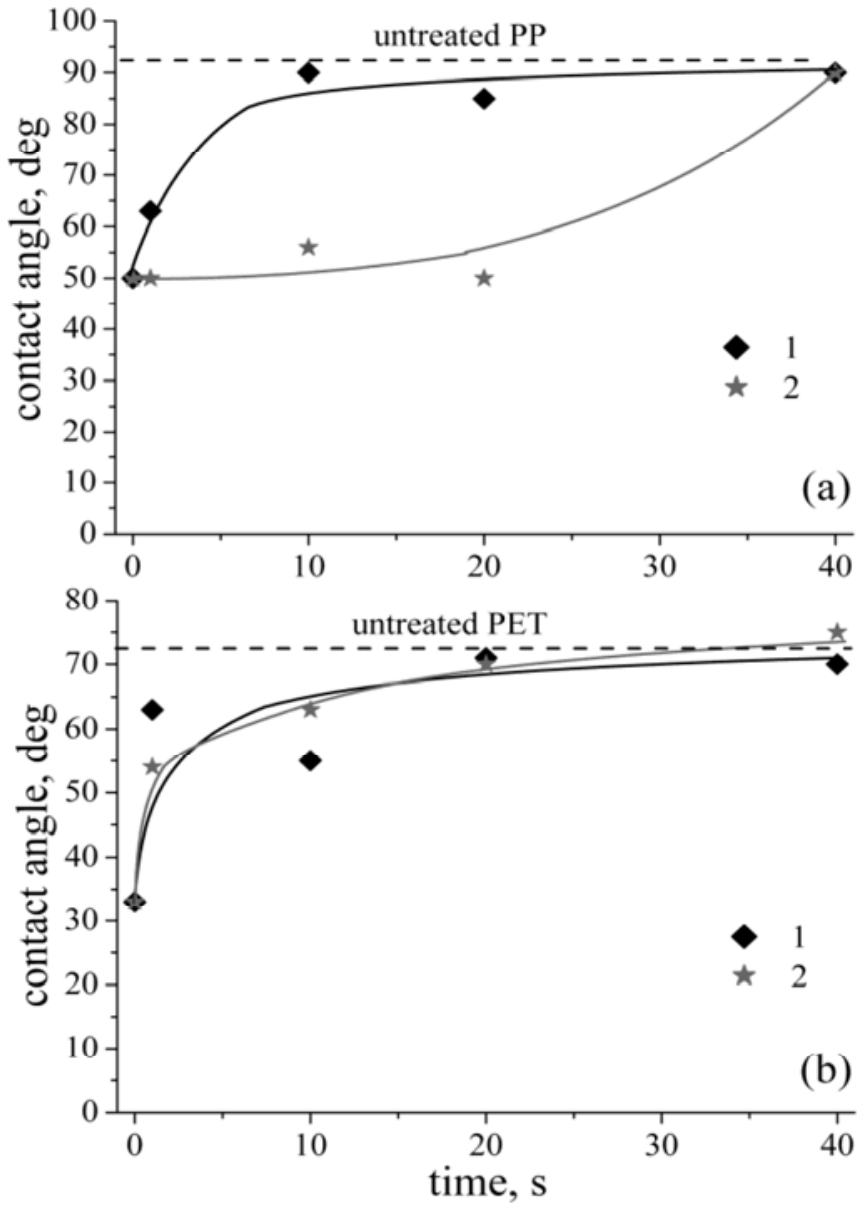

Fig. (10). The ageing of PP (a) and PET (b) films as a function of residence time in hydrogen glow discharge. The films are located on the frontal (1) side and on the back (2) side of the substrate (see Fig. 1).

now these methods are not developed for such complicated systems as NTP-modified polymer surfaces. Because of this, many scientists are forced to use semi-empirical approaches and attract different speculations in order to find a mathematical correlation between surface energy and surface parameters measured in the experiment. We also used a semi-empirical approach to describe the problem of ageing.

According to Young's equation, the cosine of the contact angle $\theta$ is related to the surface energy $\gamma_{s v}$ of the polymer film as follows: 
$\cos \theta=\frac{\gamma_{s v}-\gamma_{s l}}{\gamma_{l v}}$

where $\gamma_{l v}$ is a surface tension of the liquid, $\gamma_{s l}$ is an interfacial energy of the film-liquid interface. As a rule, the increase in surface energy of the film after plasma activation is attributed to polar functional groups (mainly oxygencontaining groups) created by NTP at the surface. We assume that the increase of surface energy of the modified film is determined not only by the amount of the bound oxygen in the surface layer but by the amount of the chemical structures containing double carbon bonds as well. Of course, each specific chemical structure (functional group) containing double carbon bond or oxygen brings its own contribution to an increase in surface energy.

For simplicity, we will use the generalized densities

$N_{C}=\frac{\int_{0}^{d} N_{C}(x) d x}{d}$

and

$N_{O}=\frac{\int_{0}^{d} N_{o}(x) d x}{d}$

of double carbon bonds and oxygen averaged over the layer; here $d$ is a modified layer thickness which determines the surface hydrophilicity. We suppose also that there is additivity in contribution of $N_{C}$ and $N_{O}$ to an additional increase in surface energy provided by NTP. In such a case, using Young's equation we can offer the relation between $\cos \theta$ and concentrations of $N_{C}$ and $N_{O}$ as follows:

$\cos \theta-\cos \theta_{1} \simeq \alpha_{C} N_{C}+\alpha_{O} N_{O}$,

where $\theta$ is the current contact angle during ageing process; $\theta_{1}$ is contact angle at the end of the first stage $\left(\theta_{1}=64.3^{\circ}\right.$ for PP and $\theta_{1}=56^{0}$ for PET); $\alpha_{C}=\beta_{C} / \gamma_{l v}$ and $\alpha_{O}=\beta_{O} / \gamma_{l v} ; \alpha_{C}, \beta_{C}$ and $\alpha_{O}, \beta_{O}$ are dimensional proportionality coefficients between additional surface energy provided by NTP and the averaged densities of double carbon bonds and oxygen in the modified layer. These coefficients are reduced to a single double bond and oxygen atom, respectively.

As mentioned above (Fig. 3), the first stage ( 10 days) in ageing of the NTP-treated films in ambient air occurs at practically constant concentration of bound oxygen in the modified surface layer. Proceeding from a large quantity of double $C=C$ bonds found in the ATR-IR spectra of the plasma treated films (Fig. 2), we assume that this stage of hydrophilicity deterioration is associated predominantly with the changes in chemical structures containing double carbon bonds. These changes lead to diminishing the number of double carbon bonds, for example, due to formation in the modified layer of cross-linking between polymer chains. The second stage of natural ageing (substantially more long-term - up to several months), most likely, is due to a gradual decrease of bound oxygen in the surface layer. It is also possible that cross-linking of polymer chains, which occurred in the first stage, strongly restricts the mobility of polymer chains which results in a great stability of functional groups containing oxygen in the second stage.

To explain the first stage of natural ageing we offer a hypothesis as follows. It is known [26] that the double $C=C$ bonds can migrate along the polymer chain. Usually, $C=C$ bonds migration takes place in the presence of the appropriate impurity-catalysts, which can drastically accelerate this process. Nevertheless, this migration is possible (though much more slowly) without a catalyst due to absorption of light energy by double bonds. As a result of the random migration, the double bonds of neighboring chains are able to come closer to each other at a distance of intermolecular interactions (thermal motion of the chains also contributes to this process) and to create a cross-linking between the chains. Random migration of the double bonds is comparable with a diffusion process.

Let $N_{C}$ and $D_{C}$ denote the averaged density of $C=C$ bonds in the modified layer and their "diffusion" coefficient, respectively. The average distance $r_{C}$ between double bonds is equal to $r_{C} \approx N_{C}^{-1 / 3}$. In this case, the characteristic time $\tau$, for which two double bonds can come closer to each other due to "diffusion" (migration), is equal to $\tau \approx 1 /\left(4 D_{C} N_{C}^{2 / 3}\right)$.

The equation describing the disappearance of double bonds and its solution can be written as follows:

$$
\begin{aligned}
& \frac{\partial N_{C}}{\partial t}=-\frac{N_{C}}{\tau}=-4 D_{C} N_{C}^{5 / 3} \\
& \frac{1}{N_{C}^{2 / 3}}=\frac{1}{N_{0 C}^{2 / 3}}+\frac{8}{3} D_{C} \cdot t
\end{aligned}
$$

or

$\frac{\partial N_{i}}{\partial t}=-a_{i} I N_{i} \equiv-b_{i} N_{i}$

where $N_{O C}$ is the averaged density of double carbon bonds immediately after NTP-treatment.

Since the first stage of natural ageing is caused mainly by disappearance of double carbon bonds, we can rewrite (2) for the first stage in such a form:

$\cos \theta-\cos \theta_{1} \simeq \alpha_{C} N_{C}$

So, using the equation (4) and relation (5) the contact angle dependence on ageing time $t$ can be obtained:

$$
\left(\frac{1}{\cos \theta-\cos \theta_{1}}\right)^{2 / 3}=\frac{1}{\left(\alpha_{C} N_{0 C}\right)^{2 / 3}}+\frac{8}{3} \frac{D_{C} N_{0 C}^{2 / 3}}{\left(\alpha_{C} N_{0 C}\right)^{2 / 3}} \cdot t
$$

Fig. (11) shows the results of mathematical processing by the expression (6) of the experimental data presented in Fig. (3) for the PP and PET hydrophilicity degradation.

As can be seen in Fig. (11), the proposed hypothesis for the first stage of natural ageing of plasma modified films agrees well enough with the experimental data. This agreement probably proves that the proposed hypothesis correctly catches the key features of the ageing process. Based on this hypothesis and experimental results, one can estimate the "diffusion" (migration) coefficients of double carbon bonds in PP and PET films at room temperature. It was found that these coefficients are close to each other and 
equal to $3 \cdot 10^{-20} \mathrm{~cm}^{2} \mathrm{~s}^{-1}$ and $1.5 \cdot 10^{-20} \mathrm{~cm}^{2} \mathrm{~s}^{-1}$ within the orderof-magnitude accuracy.

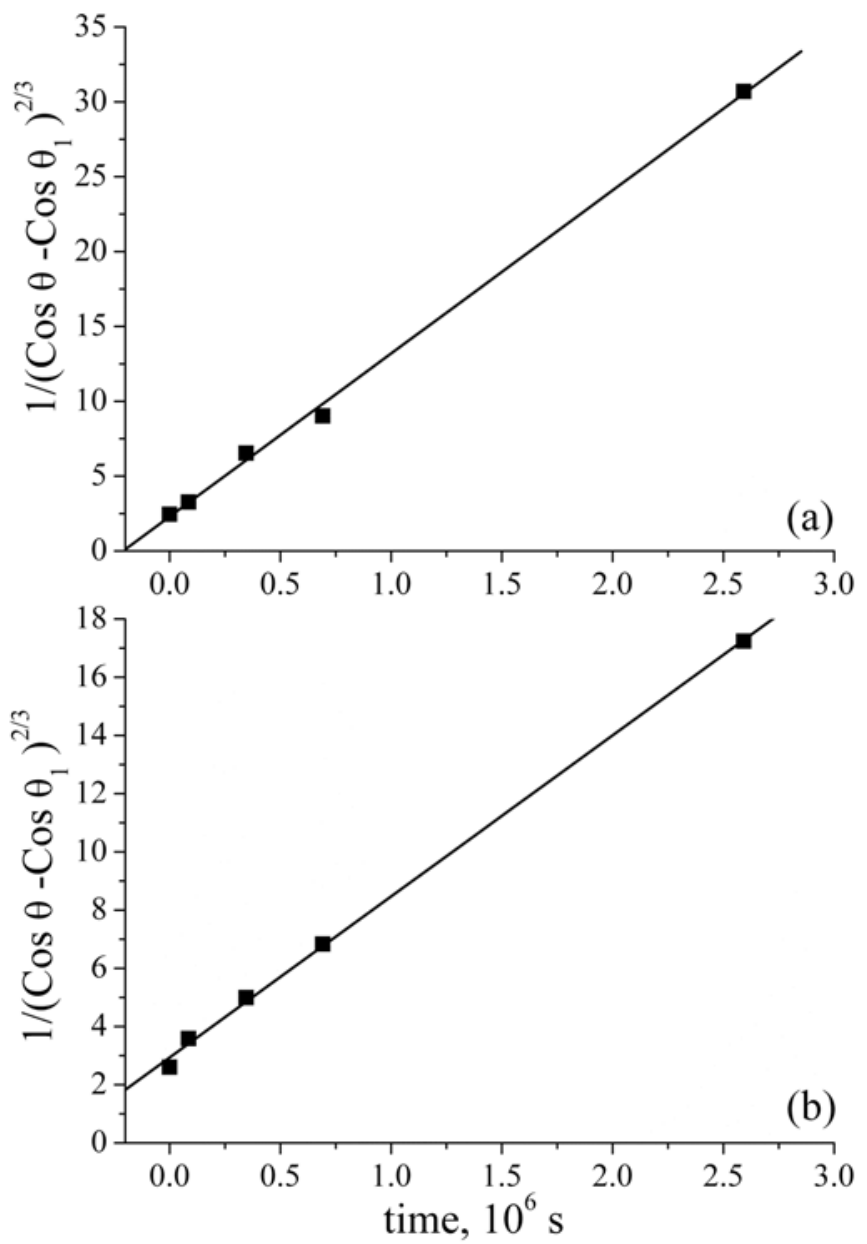

Fig. (11). The results of mathematical processing by the expression (3) of the experimental data for the natural ageing in air of the plasma modified PP (a) and PET (b) films.

\subsection{On the Accelerated Ageing Induced by UV Irradiation}

Hydrophilic films ageing induced by UV radiation in vacuum is considerably faster than ageing in air. In this case, migration of double bonds can be neglected. One can assume therefore that accelerated ageing occurs due to local processes involving predominantly photons and chemical structures with double carbon bonds and oxygen containing functional groups. There are a lot of specific photo-chemical reactions in polymers resulting in breaking of double carbon bonds and elimination of oxygen. Unfortunately, information about the rate coefficients for such reactions in solid polymers is very scarces.

We will again use the number densities of double carbon bonds $N_{C}$ and oxygen $N_{O}$ averaged over the modified layer and will assume that each of them is characterized by generalized rate coefficients reduced to the intensity of UV radiation. We assume also that absorption length of UV photons is much greater than the thickness of the modified layer. So the equation describing the disappearance of $N_{C}$ and $N_{O}$ due to absorption of UV radiation looks as follows: $\frac{\partial N_{i}}{\partial t}=-a_{i} I N_{i} \equiv-b_{i} N_{i}$,

where $N_{i}$ is a density of photoactive chemical structures of type $i$ ( $i=C$ means double carbon bonds, $i=O$ means oxygen containing groups); $I$ is the UV light intensity inside the modified layer; $a_{i}$ is a product of the photon absorption cross-section and the photo-induced reaction probability of the structure of type $i ; b_{i}^{-1} \equiv \tau_{i}$ is a characteristic "lifetime" of the structure of type $i$ in the presence of UV irradiation.

Using modified relation (2) $\left(\theta_{2}\right.$ instead of $\left.\theta_{1}\right)$ the following expression can be obtained from (7):

$\cos \theta-\cos \theta_{2} \cong \alpha_{C} N_{0 C} e^{-t b_{C}}+\alpha_{O} N_{0 O} e^{-t b_{O}}$,

where $N_{O C}$ and $N_{O O}$ are the densities of $N_{C}$ and $N_{O}$ immediately before UV irradiation; $\theta_{2}$ is contact angle close to the contact angle of the untreated film $\left(\theta_{2}=93^{\circ}\right.$ for PP and $\theta_{2}=73^{0}$ for PET).

The results of fitting the expression (8) to the experimental data from Figs. $(\mathbf{8}, \mathbf{9})$ are presented in Figs. $(12,13)$.
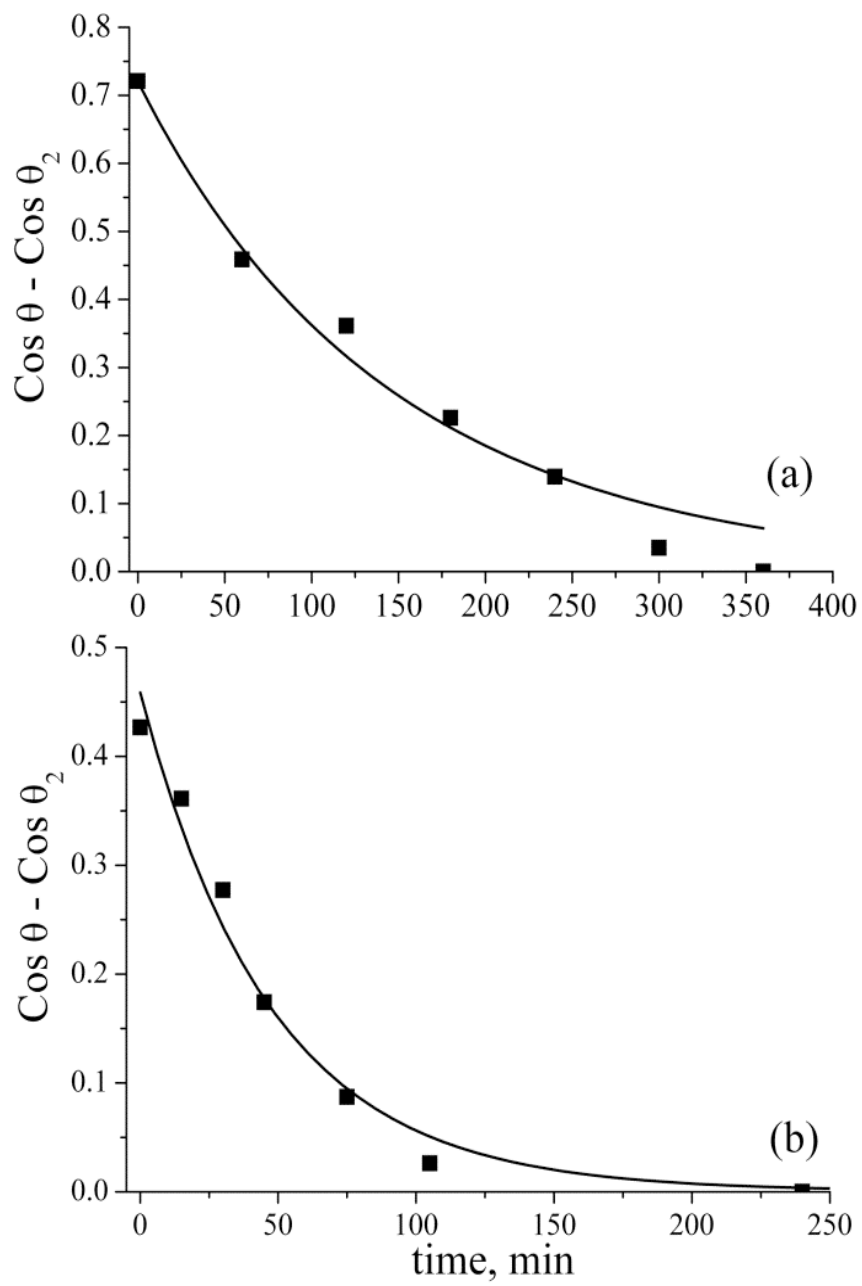

Fig. (12). The results of mathematical fitting the expression (8) to the experimental data for the ageing of hydrophilic PP films. a) Hydrophilic PP film is unwashed in ethanol; b) hydrophilic PP film is washed in ethanol. 

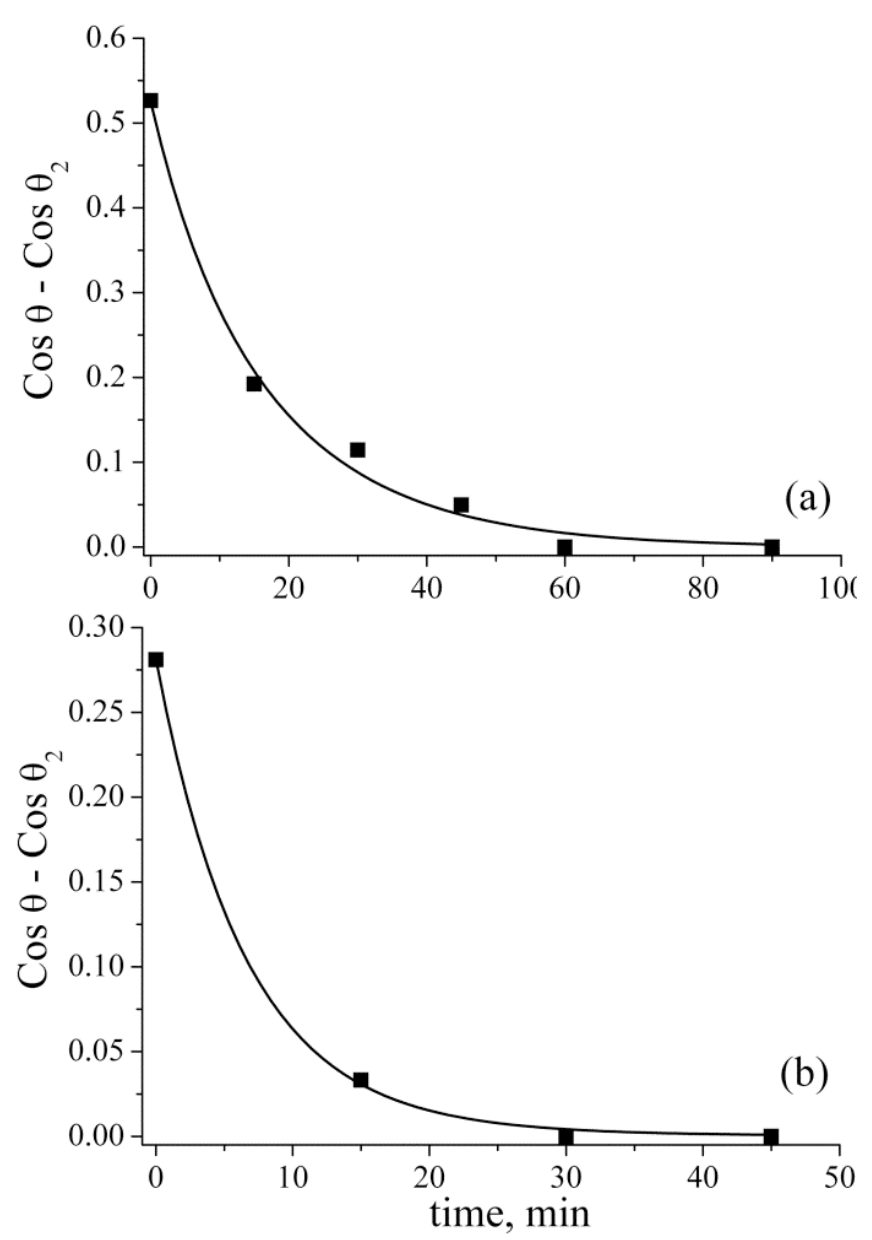

Fig. (13). The results of mathematical fitting the expression (8) to the experimental data for the ageing of hydrophilic PET films. a) Hydrophilic PET film is unwashed in ethanol; b) hydrophilic PET film is washed in ethanol.

The fitting curves describing the experimental dependences of $\cos \theta-\cos \theta_{2}$ on the time of UV irradiation of hydrophilic PP and PET films are as follows (time in minutes):

(data for PP films):

a) $\cos \theta-\cos \theta_{2} \cong 0.7 e^{-t / 150}+0.02 e^{-t / 47}$

(upper loosen layer is not removed)

b) $\cos \theta-\cos \theta_{2} \cong 0.004 e^{-t / 50}+0.45 e^{-t / 47}$

(upper loosen layer is removed) (data for PET films):

a) $\cos \theta-\cos \theta_{2} \cong 0.46 e^{-t / 18}+0.06 e^{-t / 6.5}$

(upper loosen layer is not removed)

b) $\cos \theta-\cos \theta_{2} \cong 0.08 e^{-t / 18}+0.27 e^{-t / 6.5}$

(upper loosen layer is removed)

The multipliers in front of exponents are proportional to the number densities of $N_{C}$ and $N_{O}$. So the obtained results can be interpreted as follows. In the upper loosen layer of the modified film the number of the structures with double carbon bonds (see coefficients 0.7 and 0.46 for unwashed PP and PET films) prevails over the amount of bound oxygen (see coefficients 0.02 and 0.06 for unwashed PP and PET films). However, the density of structures with $C=C$ bonds drops sharply down into the interior of the polymer film (see coefficients 0.004 and 0.08 for washed PP and PET films), so that after removal of the loosen layers the chemical structures containing oxygen dominate in the modified layer (see coefficients 0.45 and 0.27 for washed PP and PET films). Qualitative illustration on spatial structure of the modified layer is given in Fig. (14).

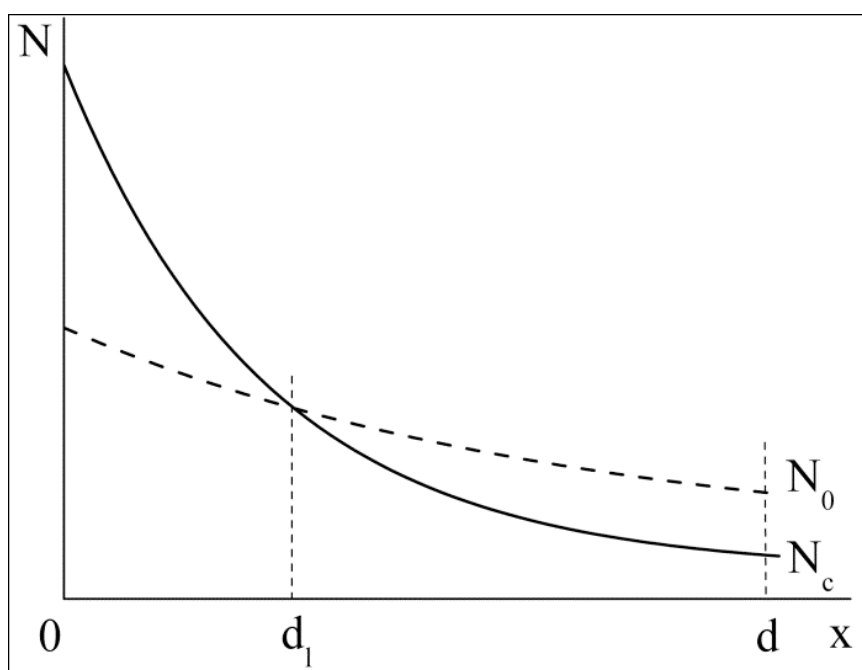

Fig. (14). Qualitative illustration on spatial distribution of $N_{C}$ and $N_{O}$ in depth of the modified layer of a polymer film. Here $d_{l}$ is the thickness of loosen layer; $d$ is total thickness of NTP-modified layer.

In the hydrophilic PP and PET films irradiated by UV photons, the characteristic "lifetime" of double carbon bonds (150 and $18 \mathrm{~min}$ for PP and PET respectively) is approximately 3 times higher than the "lifetime" of bound oxygen (47 and 6.5 min respectively). The PET "lifetimes" of double carbon bonds $(18 \mathrm{~min})$ and bound oxygen $(6.5$ min) are approximately 7 times less than those for PP films (150 and $47 \mathrm{~min}$ respectively). We attribute this effect to the difference in the chemical structures of PP and PET films. Therefore, PET absorbs UV radiation not only by plasma created chromophore groups but by own chromophore groups as well.

Based on these results, we estimated the average values of products of the reaction quantum efficiency and the absorption cross-section of UV quantum for the structures containing double carbon bonds and oxygen containing groups: $10^{-20} \mathrm{~cm}^{2}$ and $3 \cdot 10^{-20} \mathrm{~cm}^{2}$ for PP, and $7 \cdot 10^{-20} \mathrm{~cm}^{2}$ and $2 \cdot 10^{-19} \mathrm{~cm}^{2}$ for PET respectively.

\section{CONCLUSIONS}

It is experimentally established that degradation of hydrophilicity of the NTP-modified PP and PET films accelerates significantly by ultraviolet irradiation with a wavelength $\lambda=253.7 \mathrm{~nm}$, and the contact angle behavior depends on both the film type and the environment (vacuum or air), where the films are processed by UV irradiation. PP and PET films processing by hydrogen atoms has an even stronger influence (compared to UV) on the loss of their 
hydrophilic properties. In this case, the hydrophilicity degradation time reduces to $10-40 \mathrm{~s}$.

The obtained results indicate that numerical simulation of polymer film hydrophilicity ageing must take into consideration in the modified layer not only the elimination of the bound oxygen from the oxygen containing functional groups, but disappearance of the chemical structures with double carbon bonds as well.

\section{CONFLICT OF INTEREST}

The authors confirm that this article content has no conflict of interest.

\section{ACKNOWLEDGEMENTS}

The authors thank Prof. A. B. Gilman for helpful discussions.

This work was partially supported by the Russian Foundation for Basic Research (project № 08-02-00601-a).

\section{REFERENCES}

[1] Gilman AB, Drachev AI, Kuznetsov AA, Lopukhova GV, Potapov VK. Kinetic characteristics of dissociation of oxygen molecules in the positive column of a direct-current discharge. High Energy Chem 1997; 31: 121-5.

[2] Akishev Yu, Leys C, Temmerman E, Trushkin N, Versshuren J. Surface modification with a remote atmospheric pressure plasma: DC glow discharge and surface streamer regime. J Phys D Appl Phys 2005; 38: 505-9.

[3] Ponomarev AN, Vasilets VN, Talroze RV. Applications of low temperature plasma (survey). Russ Chem Phys Rep 2002; 21: 96102. (in Russian).

[4] Hubert R, Massino P, Guy B, Eds. Plasma Technology for Hyperfunctional Surface. Food, Biomedical and Textile Applications. Weinheim: WILEY-VCH, Verlag 2010

[5] Dorai R, Kushner M. A model for plasma modification of polypropylene using atmospheric pressure discharges. J Phys D Appl Phys 2003; 36: 666-85.

[6] Akishev Yu, Grushin M, Dyatko N, et al. Studies on cold plasmapolymer surface interaction by example of PP- and PET-films. J Phys D Appl Phys 2008; 41: 235203.

[7] Zaikov GE. Ageing and stabilisation of polymers. Russ Chem Rev 1991; 60: 1145-62.

[8] Rabek JF. Polymer photodegradation mechanisms and experimental methods. London: Chapman \& Hall 1995.

[9] Hollander A, Thome J. Degradation and stability of plasma polymers. In: Plasma polymer films. London: Imperial College Press 2004; pp. 247-78.
[10] Siow KS, Britcher L, Kumar S, Griesser HJ. Plasma methods for the generation of chemically reactive surfaces for biomolecule immobilization and cell colonization - a review. Plasma Process Polym 2006; 3: 392-18.

[11] Hill JM, Karbashewski E, Lin A, Strobel M, Walzak MJ. Effects of aging and washing on UV and ozone-treated poly(ethylene terephthalate) and polypropylene. J Adhes Sci Technol 1995; 9: 1575-91.

[12] Strobel M, Lyons CS, Strobel JM, Kapaun RS. Analysis of aircorona-treated polypropylene and poly(ethylene terephthalate) films by contact-angle measurements and X-ray photoelectron spectroscopy. J Adhes Sci Technol 1992; 6: 429-43.

[13] Strobel JM, Strobel M, Lyons CS, Dunatov C, Perron SJ. Aging of air-corona-treated polypropylene film. J Adhes Sci Technol 1991; 5: 119-30.

[14] Clark DT, Dilks A. ESCA applied to polymers. XVIII. RF glow discharge modification of polymers in helium, neon, argon, and krypton. J Polym Sci Pol Chem [A] 1978; 16: 911-36.

[15] Shahidzadeh-Ahmadi N, Arefi-Khonsari F, Amouroux J. Characterization of the acid-base properties of $\mathrm{NH}_{3}$ or $\mathrm{O}_{2}$ plasmatreated polypropylene films and the effects of ageing. J Mater Chem 1995; 5: 229-36.

[16] Hong J, Truica-Marasescu F, Martinu I, Wertheimer MR. An investigation of plasma-polymer interactions by mass spectrometry. Plasmas Polym 2002; 7: 245-60.

[17] Wilken R, Hollander A, Behnisch J. Vacuum ultraviolet photolysis of polyethylene, polypropylene and polystyrene. Plasmas Polym 2002; 7: 19-39.

[18] Gilman AB, Rishina LA. Structural transformations in the bulk of polypropylene upon plasma treatment. High Energy Chem 2003; 37: 291-6.

[19] Wilken R, Hollander A, Behnish J. Quantitative comparison between vacuum-ultraviolet irradiation and remote hydrogen plasma treatment of hydrocarbon polymers. Plasmas Polym 1998; 3: 165-75.

[20] Akishev Yu, Napartovich A, Trushkin N, Grushin M. Novel AC and DC non-thermal plasma sources for cold surface treatment of polymer films and fabrics at atmospheric pressure. Plasmas Polym 2002; 7: 261-89.

[21] Niyuzi F, Savenkova IV. New concepts in polymer science. Photodegradation and light stabilization of heterochain polymers. Boston: VSP, Leiden 2006.

[22] Allen NS, Ed. Handbook of photochemistry and photophysics of polymer materials. Hoboken: Wiley \& Sons Inc 2010.

[23] Wertheimer MR, Fozza AC, Hollander A. Industrial processing of polymers by low-pressure plasmas: the role of VUV radiation. Nucl Instrum Methods Phys Res B 1999; 151: 65-75.

[24] Paynter RW. XPS studies of the ageing of plasma-treated polymer surfaces. Surf Interface Anal 2000; 29: 56-64.

[25] Occihiello E, Morra M, Morini G, Garbassi F. Oxygen-plasmatreated polypropylene interfaces with air, water, and epoxy resins: Part I. Air and water. J Appl Polym Sci 1991; 42: 551-9.

[26] Ingold CK. Structure and Mechanism in Organic Chemistry. New York: Cornell University Press 1969. 